University of Wollongong

Research Online

Faculty of Engineering and Information

Faculty of Engineering and Information

Sciences - Papers: Part A

Sciences

$1-1-2012$

Experimental investigation of a hydraulically interconnected suspension in vehicle dynamics and stability control

Lifu Wang

University of Technology, Sydney

Nong Zhang

University of Technology, Sydney

Haiping Du

University of Wollongong, hdu@uow.edu.au

Follow this and additional works at: https://ro.uow.edu.au/eispapers

Part of the Engineering Commons, and the Science and Technology Studies Commons

Research Online is the open access institutional repository for the University of Wollongong. For further information contact the UOW Library: research-pubs@uow.edu.au 


\title{
Experimental investigation of a hydraulically interconnected suspension in vehicle dynamics and stability control
}

\author{
Abstract \\ Mainly motivated by developing cost-effective vehicle anti-roll systems, hydraulically interconnected \\ suspension has been studied in the past decade to replace anti-roll bars. It has been proved theoretically \\ and practically that hydraulic suspensions have superior anti-roll ability over anti-roll bars, and therefore \\ they have achieved commercial success in racing cars and luxury sports utility vehicles (SUVs). However, \\ since vehicle is a highly coupled complex system, it is necessary to investigate/evaluate the hydraulic- \\ suspension-fitted-vehicle's dynamic performance in other aspects, apart from anti-roll ability, such as ride \\ comfort, lateral stability, etc. This paper presents an experimental investigation of a SUV fitted with a \\ hydraulically interconnected suspension under a severe steady steering maneuver; the result is compared \\ with a same type vehicle fitted with anti-roll bars. Furthermore, an insight of how the hydraulic suspension \\ response to extreme maneuvers has been presented, which is of great interest to the optimization of the \\ hydraulic suspension design. Real time simulations are used to assist the experiment to provide a \\ complete picture of the hydraulic system response. The findings are discussed.
}

\section{Keywords}

experimental, hydraulically, interconnected, suspension, investigation, control, stability, vehicle, dynamics

\author{
Disciplines \\ Engineering | Science and Technology Studies
}

\section{Publication Details}

L. Wang, N. Zhang \& H. Du, "Experimental investigation of a hydraulically interconnected suspension in vehicle dynamics and stability control," SAE International Journal of Passenger Cars: Mechanical Systems, vol. 5, (2) pp. 759-768, 2012. 


\title{
Experimental Investigation of a Hydraulically Interconnected Suspension in Vehicle Dynamics and Stability Control
}

\author{
Lifu Wang and Nong Zhang \\ University of Technology, Sydney \\ Haiping Du \\ University of Wollongong
}

\begin{abstract}
Mainly motivated by developing cost-effective vehicle anti-roll systems, hydraulically interconnected suspension has been studied in the past decade to replace anti-roll bars. It has been proved theoretically and practically that hydraulic suspensions have superior anti-roll ability over anti-roll bars, and therefore they have achieved commercial success in racing cars and luxury sports utility vehicles (SUVs). However, since vehicle is a highly coupled complex system, it is necessary to investigate/evaluate the hydraulic-suspension-fitted-vehicle's dynamic performance in other aspects, apart from anti-roll ability, such as ride comfort, lateral stability, etc. This paper presents an experimental investigation of a SUV fitted with a hydraulically interconnected suspension under a severe steady steering maneuver; the result is compared with a same type vehicle fitted with anti-roll bars. Furthermore, an insight of how the hydraulic suspension response to extreme maneuvers has been presented, which is of great interest to the optimization of the hydraulic suspension design. Real time simulations are used to assist the experiment to provide a complete picture of the hydraulic system response. The findings are discussed.
\end{abstract}

CITATION: Wang, L., Zhang, N. and Du, H., "Experimental Investigation of a Hydraulically Interconnected Suspension in Vehicle Dynamics and Stability Control," SAE Int. J. Passeng. Cars - Mech. Syst. 5(2):2012, doi:10.4271/2012-01-0240.

\section{INTRODUCTION}

Suspension systems play key roles in road vehicles to ensure an energy-optimal, safe and comfortable driving. Nowadays the dominant suspensions on the market are still passive systems, due to not only their cost effectiveness but also reliabilities. The design of passive independent wheel suspension is a process of compromising, since four wheel vehicles have four suspension modes: bounce, roll, pitch, and warp $[\underline{1}, \underline{2}, \underline{3}, \underline{4}]$, and they do not share the same preference of stiffness and damping. For example, a soft bounce mode is appreciated by ride comfort, while stiff roll and pitch modes are beneficial for inhibiting vehicle attitude during steering, braking, and acceleration. On the other hand, suspension warp mode should be as soft as possible for road-holding performance.

To overcome these compromises, interconnected suspensions have been adopted as one solution to achieve the vehicle dynamic performance in a particular mode, e.g., increasing stiffness in roll or pitch mode. A good example is anti-roll bars (stabilizer bars), mechanically interconnected suspension systems, employed to increase suspension stiffness in roll mode. However, since anti-roll bars are not fully uncoupled systems, they bring side effects. For instance, anti-roll bars will undesirably stiffen the suspension warp mode which weaken road holding, especially during off-road drive. One example of the commercial solution to this problem is Toyota's Kinetic Dynamic Suspension System (KDSS), which can effectively disengage anti-roll bars during off-road drive. Another issue with anti-roll bars is that damping can hardly be added in accompanying with the increased torsional stiffness in vehicle roll plane.

Moving from suspension level up to whole vehicle dynamics level, more motions are coupled with those four sprung mass dominated suspension modes to some extends, such as vehicle lateral and yaw motions, which are nursed by/ coupled together with suspension warp and roll modes. Firstly, these couplings offer us a great opportunity to indirectly control vehicle lateral/yaw motion by controlling one or a set of according suspension modes. For example, Mercedes Corsswind Assistant demonstrates an indirect control of vehicle yaw through suspension warp mode [5]. The suspension warp mode is generated by pushing diagonal

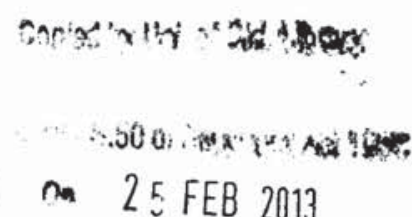


wheels to the ground through $\mathrm{ABC}$ active suspension, which leads to a resulted yaw moments.

Secondly, due to the coupling of vehicle motions and suspension modes, in order to evaluate an anti-roll system, it is necessary to investigate the effects that it brought to other vehicle motions. Hydraulically Interconnected Suspension (HIS) is a low cost, effective anti-roll suspension system. Its anti-roll ability and performance has been studied through analysis and experiment []. However, the experimental investigation of HIS in vehicle vertical and lateral motions has not been carried out in field tests yet. This paper presented the experimental study of a vehicle fitted with HIS in roll, vertical and lateral aspects, while using a standard vehicle configured with anti-roll bars as comparison. The paper is organized in the following way. First, a brief background of hydraulically interconnected suspensions is presented, followed by the modeling of a vehicle and a HIS system. Then the field tests are outlined and the obtained experimental results are reported. Simulations are used to further explore the system and guide the experiment.

\section{HYDRAULICALLY INTERCONNECTED SUSPENSIONS}

Interconnected suspensions are mainly motivated by the desire of a stiffer vehicle roll mode. A passive interconnected suspension system is one in which the motion (displacement, velocity) at one wheel station can produce forces at other wheel stations, which is generally realized through either mechanical or fluidical means. Interconnected suspensions in a full car level have the theoretically potential ability to uncouple the four suspension modes: roll, bounce, pitching and articulation $[\underline{1} ; \underline{4}]$, and have advantages in adjusting stiffness/damping in each of these modes. A comprehensive survey on recent suspension development is presented with a focus on interconnected suspensions [7], and in [8] a survey of passive interconnected suspension has been carried out.

Let's start with mechanically interconnected suspensions. The full-vehicle mechanically interconnected suspensions have been developed and investigated for many years []ㅡ, which could decouple the different suspension modes, in order to provide a more favourable compromise between ride and handling requirements. However, their designs are complex and the added weights are considerable. Besides, they are difficult to be tuned to adapt various road and operating conditions.

Unlike mechanical interconnected suspensions which are heavy and difficult to offer additional damping, fluidic suspension systems can offer viable options in improving mode properties. The fluidical interconnection can be realized through hydraulic fluids, pneumatic fluids, or a combination of those two. Full-vehicle fluidically interconnected suspension systems have been investigated on the topics of pneumatically interconnected suspensions [9], hydraulically interconnected suspensions (HIS), $[8 ; \underline{10}, \underline{11}, 12,13]$, hydropneumatic suspensions $[\underline{2} ; \underline{14}, \underline{15}, \underline{16}, \underline{17}]$, and interconnection configuration $[1 ; 18]$. Variable fluidically interconnected suspensions are developed for different applications.

Cao etc. $[2 ; 14 ; 15 ; \underline{19}]$ investigated the dynamics of interconnected hydro-pneumatic suspensions at a full car level, particularly for heavy vehicle applications. The hydro. pneumatic suspension systems have been employed in heavy military vehicles for nearly half a century. The fluidic couplings are realised through hydro-pneumatic struts [1]; 20], which have compact design and are claimed to have larger effective working area than normal hydraulic cylinders. $z_{W}$ The struts provide considerable flexibility for various interconnection configurations among the hydraulic and pneumatic chambers, either hydraulically or pneumatically $Z_{i_{3}}$ [19]. A general framework for designing and tuning is included in $[2 ; 14]$.

Hydraulically interconnected suspensions have achieved commercial success, e.g., Kinetic $\mathrm{H} 2$ suspensions, which are originally developed by Australian company Kinetic Pty Ltd. Kinetic $\mathrm{H} 2$ suspensions typically contain four double-acting hydraulic cylinders in addition to the original vehicle suspensions. The cylinders are mounted on the car body and the piston rods are fixed on the wheel assemblies. The chambers in the cylinders are interconnected by hydraulic circuits, arranged to counter vehicle roll motion. Each circuit comprises elements such as damper valves, hydraulic accumulators, pipelines, fittings and flexible hoses. Kinetic Dynamic Suspension System (KDSS) is a successful commercial application of this kind of technology, and different versions are available in several applications, e.g. Lexus GX 470 and 200 Series Toyota Land Cruiser. The performance is reported in $[\underline{13} ; \underline{21}]$.

Zhang \& Smith $[\underline{8} ; \underline{10}, \underline{11}, \underline{12}]$ recently studied the dynamics of vehicles fitted with HIS systems with a focus on the multi-body system dynamics and interconnected fluid circuit dynamics. A systematic approach are proposed for studying hydraulically interconnected suspensions in both time and frequency domain. The finite element modeling of nonlinear hydraulic system is seamlessly connected to a mass-spring vehicle model through hydraulic-mechanical kinetic coupling, and the theoretical analyses are validated by laboratory experiments [22]. This paper further extended this research to study the transient responses of a typical SUV fitted with a HIS through both simulations and field tests.

\section{MODELING}

A brief description of the HIS is provided here in order to give a complete background of the system dynamics. A lumped-mass 9 degrees-of-freedom (DOF) full-car model, as shown in Figure 1, is used in this study. The modeling details of a vehicle fitted with an HIS have been included in [22]. 
ted

of

car

Iro-

avy

idic

[17;

tave

lers.

ious

and

zally

$\mathrm{g}$ is

eved

h are

Ltd.

cting

hicle

$y$ and

The

raulic

sircuit

raulic

inetic

:essful

', and

;, e.g.,

r. The

:d the cus on 1 fluid ied for $n$ both ling of $d$ to a hanical lated by ded this al SUV sts.

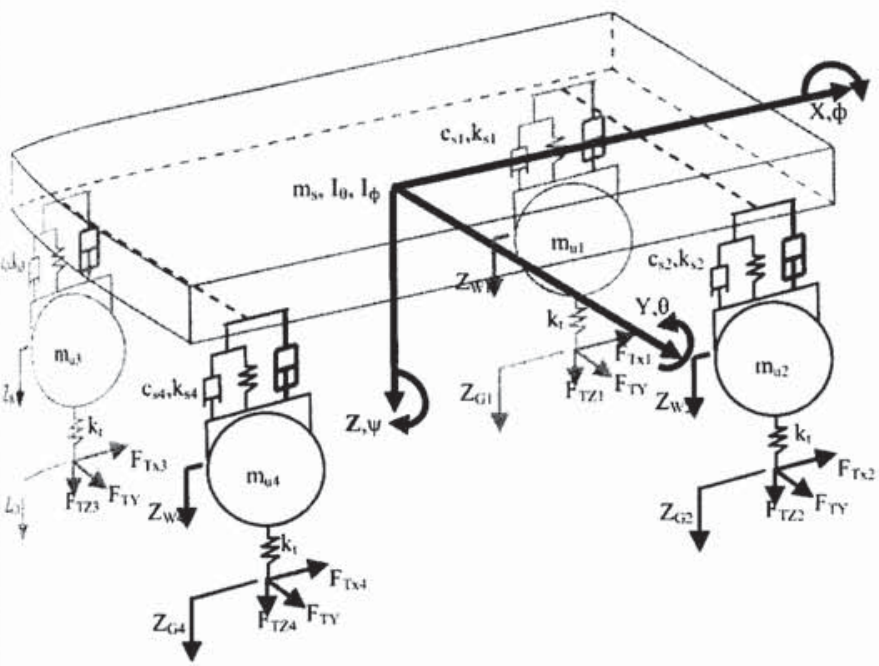

Figure 1. Schematic of a full-car with an HIS

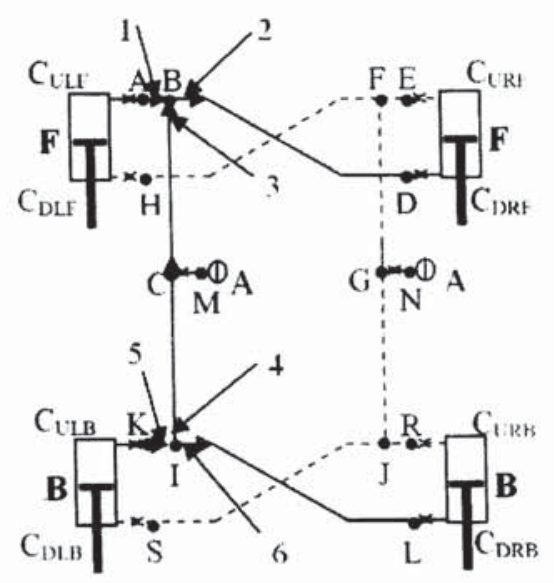

Figure 2. Schematic of a roll-resistant HIS

The vehicle suspension system consists of linear tire damping and springing, linear conventional suspension springing, and a typical HIS system. The car body sprung mass is considered to have five DOF and each of the four wheel unsprung masses has only one DOF in vertical direction. Magic-Formula tire models are used to calculate the contact forces. From Figure 1, it can be seen that the shock absorbers of conventional independent suspensions between the sprung and unsprung masses are kept with the car. The added typical roll-resistant HIS arrangement for this application consists of two identical hydraulic circuits as shown in Figure 2. The two hydraulic circuits are coupled with each other kinetically via four identical double acting piston-cylinder actuators. Each of the hydraulic circuits comprises a nitrogen-filled diaphragm accumulator, and bydraulic pipeline elements. The HIS circuits often include additional elements, such as hydraulic fittings and flexible hoses, but they fall beyond the consideration of this investigation.
Within the two hydraulic circuits, the cylinders are mounted on the car body and the piston rods are fixed on the wheel stations. The dynamic interaction between the hydraulic system and the sprung and unsprung masses can be described as such: relative velocities in the suspension struts cause the changes of pressure in both circuits, which leads to the changes of suspension strut forces through four cylinders. As a result, vehicle body and wheel motions occur, which, in turn, affect the hydraulic system. This mechanic-hydraulic interaction will continue until the system reaches a new equilibrium. HIS systems can provide greater freedom to independently specify modal stiffness and damping characteristics. The working mechanisms and features of the full-car HIS system can be found in [12].

\section{STATE SPACE EQUATIONS}

The details of derivation of the state space equations of the combined vehicle multi-body system and hydraulic circuits for transient analysis can be found in [12]. Here a brief description of the dynamic model is provided, including the state space representation of the integrated full-car system for the transient analysis. The state vector describing the motion of the sprung and unsprung lumped multi-body subsystem is defined as

$$
X_{M}=\left[\begin{array}{ll}
Z^{T} & \dot{Z}^{T}
\end{array}\right]^{T}
$$

where displacement vector is

$$
Z=\left[\begin{array}{lllllllll}
Y_{\mathrm{v} 0} & Z_{\mathrm{v} 0} & Z_{\mathrm{w} 1} & \mathrm{Z}_{\mathrm{w} 2} & \mathrm{Z}_{\mathrm{w} 3} & \mathrm{Z}_{\mathrm{w} 4} & \varphi_{\mathrm{xv}} & \theta_{\mathrm{Yv}} & \psi_{\mathrm{ZV}}
\end{array}\right]^{T}
$$

and velocity vector is

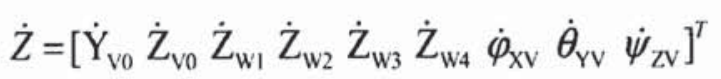

The state vector describing the dynamic sates of the hydraulic subsystem is defined as

$$
\begin{aligned}
X_{H}= & {\left[P_{U}^{C 1} P_{A}^{A B} P_{B}^{A B} Q_{B}^{A B} P_{M}^{A B M} P_{C}^{B C} Q_{C}^{B C} Q_{C}^{C l} P_{I}^{C l} Q_{l}^{C I} \ldots\right.} \\
& Q_{I}^{I l} P_{L}^{I L} P_{B}^{C 4} P_{U}^{C 2} P_{E}^{E F} P_{F}^{E F} Q_{F}^{E F} P_{N}^{A F N} P_{G}^{F G} Q_{G}^{F G} Q_{G}^{G J} \ldots \\
& P_{J}^{(i)} Q_{J}^{G J} Q_{J}^{J S} P_{S}^{J S} P_{B}^{C 3} P_{D}^{C D} P_{B}^{C 2} P_{U}^{C 3} P_{K}^{K I} P_{H}^{G H} P_{B}^{C 1} \ldots \\
& \left.P_{U}^{C 4} P_{R}^{R J}\right]^{T}
\end{aligned}
$$

Through integrating the two state vectors together, the state vector of the full car with a HIS is obtained

$$
X=\left[X_{M}^{T} X_{H}^{T}\right]^{\mathrm{T}} \quad(18+34=52 \text { elements })
$$


Using free body diagram approach and applying Newton's second law, the full car system state space equation is derived

$$
T \dot{X}=S X+F,
$$

$$
\left[\begin{array}{ccc}
I_{9} & 0 & 0 \\
0 & M_{9} & 0 \\
0 & 0 & \left(T_{H}\right)_{34}
\end{array}\right]\left[\begin{array}{c}
\dot{Z} \\
\ddot{Z} \\
\dot{X}_{H}
\end{array}\right]=\left[\begin{array}{ccc}
0 & I_{9} & 0 \\
-K & -C & 0 \\
0 & 0 & S_{H}
\end{array}\right]_{52 X 52}\left[\begin{array}{c}
Z \\
\dot{Z} \\
X_{H}
\end{array}\right]_{52}+\left[\begin{array}{c}
0 \\
F_{M} \\
F_{H}
\end{array}\right]_{52}
$$

The external applied forces $F_{M} \& F_{H}$ include interactive elements between hydraulic or mechanical subsystems. They can be separated into

$$
\begin{gathered}
F_{M}=F_{m}+S_{H 2 M} X_{H} \\
F_{H}=F_{h}+K_{Z 2 H} Z+C_{\dot{Z} 2 H} \dot{Z}
\end{gathered}
$$

That is:

$$
\left[\begin{array}{ccc}
I_{9} & 0 & 0 \\
0 & M_{9} & 0 \\
0 & 0 & \left(T_{H}\right)_{34}
\end{array}\right]\left[\begin{array}{c}
\dot{Z} \\
\ddot{Z} \\
\dot{X}_{H}
\end{array}\right]=\left[\begin{array}{ccc}
0 & I_{9} & 0 \\
-K & -C & S_{H 2 M} \\
K_{Z 2 H} & C_{Z / 2 H} & S_{H}
\end{array}\right]_{S 2 X 32}\left[\begin{array}{c}
Z \\
\dot{Z} \\
X_{H}
\end{array}\right]_{52}+\left[\begin{array}{c}
0 \\
F_{m} \\
F_{h}
\end{array}\right]_{52}
$$

where $\mathbf{M}, \mathbf{K}, \mathbf{C}$ are the mass, stiffness and damping coefficient matrices respectively; $S_{H 2 M}, K_{Z 2 H}, C_{Z_{2} H}$ are the coefficients matrix coupling the motions of the lumped mechanical system and dynamics of the suspension fluid circuits; $S_{H}$ is the coefficient matrix determining the dynamics of the fluid circuits.

To obtain the solution of Equation (10), the dynamic state of the multi-body system and state of the fluid circuits can be determined simultaneously using the first 18 equations for the multi-body system and the last 34 equations for the fluid circuits respectively. The following provides a brief description of the modeling and the numerical solution scheme of the fluid circuit dynamics. The assumptions and discussions on the solution scheme can be found in $[10 ; 11]$.

\section{FIELD TEST}

A hydraulically interconnected suspension system was designed and assembled onto a typical SUV vehicle in the Dynamics Laboratory of University of Technology, Sydney, as shown in Figure 3. This experimental vehicle is a 2004 Ford Territory, shown in Figure 6. The anti-roll bars were taken off from this vehicle and replaced by the HIS system. The HIS system is pre-charged with a certain pressure, e.g., $20 \mathrm{bar}, 40 \mathrm{bar}$, etc. The higher the pre-charged pressure is, the stiffer the roll stiffness it brings to the vehicle, vice verse.
When the pre-charged pressure is atmosphere pressure, i.e., Obar, the HIS system can be treated as completely defunctioned with ignorable stiffness. The testing data from Obar HIS test can be used as benchmark of the vehicle with no anti-roll systems. A series of tests is conducted under same test condition to verify the effectiveness of the HIS system on the experimental vehicle, with different HIS precharged pressure settings. Furthermore, in Figure 7, a same year/model vehicle with standard configuration, i.e., anti-roll bars, is used to conduct the same test and to compare with the experimental vehicle.

The sensing system includes a LabJack data acquisition board, a laptop with Labview, and 8 transducers. The board is attached to the rear seats of the car during tests, as shown in Figure 5. Transducers include four accelerometers, two linear displacement transducers and two pressure transducers. Two accelerometers are installed underneath the storage box between the two front seats, which is at the CG (center of gravity) of the vehicle, to measure the lateral and longitude acceleration respectively. Other two accelerometers are installed onto the left and right chassis edges in the roll plane that crosses the CG point, vertically mounted. Taking the average value of these two sensors is the vertical acceleration of the vehicle while taking the subtraction of them can estimate the vehicle roll angular acceleration. Two displacement transducers are used to measure the suspension travel in front and two pressure sensors are installed to monitor the hydraulic system at point $\mathrm{A}$ and $\mathrm{E}$, referring to Figure 2.

The overall test plan includes roll, pitch and bounce tests. Roll tests include slalom tests and round about tests. Slalom tests are dynamic maneuvering tests and the result has been summarized in [ $\underline{6}]$. Round about tests are steady maneuvering tests with a constant steering angle and vehicle speed. Steady maneuvering generates a constant lateral acceleration to the vehicle body to excite body roll, and this lateral acceleration should be adequately large. Ideally to ensure safety and eliminate human errors, testing vehicles should be self-driven in a constant high speed, maneuvered by an automatic steering mechanism to conduct the pre-designed maneuver pattern, which is however not available in this case.

Hence, to obtain a large lateral acceleration and also ensure the safety of testing personal, a testing plan at lower speed but with larger steering angle is strategically adopted for risk management purpose. As a result, as shown in Figure $\underline{8}$, the testing vehicle driven by an experienced driver entered a roundabout circular path of $8 \mathrm{~m}$ radius and exited after a 540 degree clockwise turning with constant steering angle at a speed of $25 \mathrm{~km} / \mathrm{h}$. The lateral acceleration measured from these tests reached $0.7 \mathrm{~g}$ which is sufficient according to literature most vehicles tested for handling evaluations experience a lateral acceleration up to $0.8 \mathrm{~g}$. [23; 24] . 


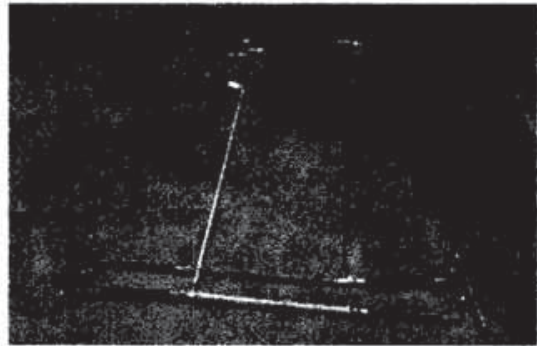

Figure 3. The assembled HIS system

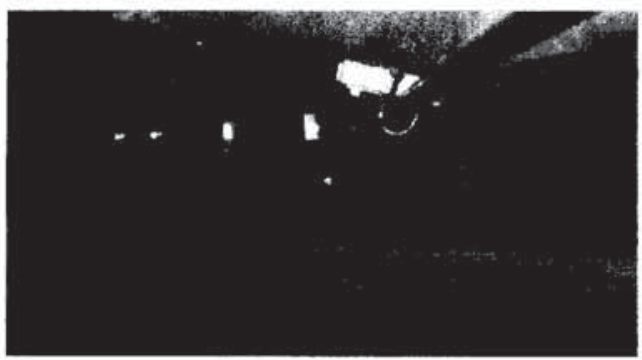

Figure 4. Mounting of the accumulator

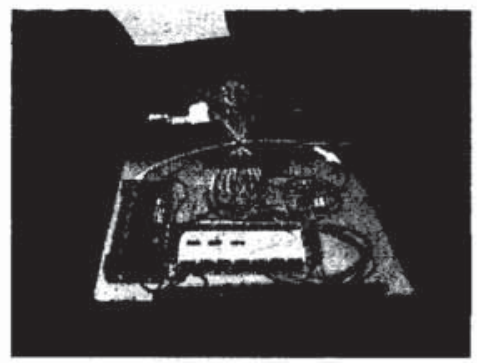

Figure 5. Data acquisition board

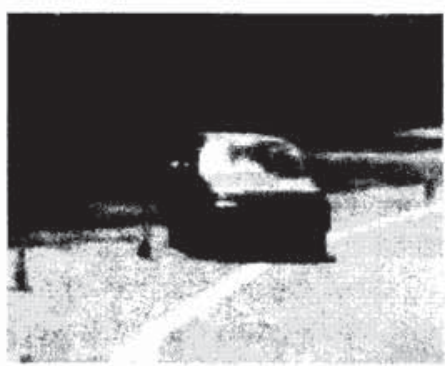

Figure 6. Experiment vehicle

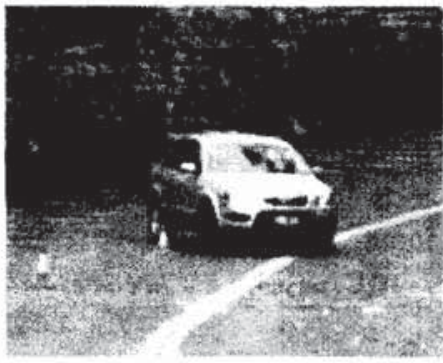

Figure 7. Contrast vehicle

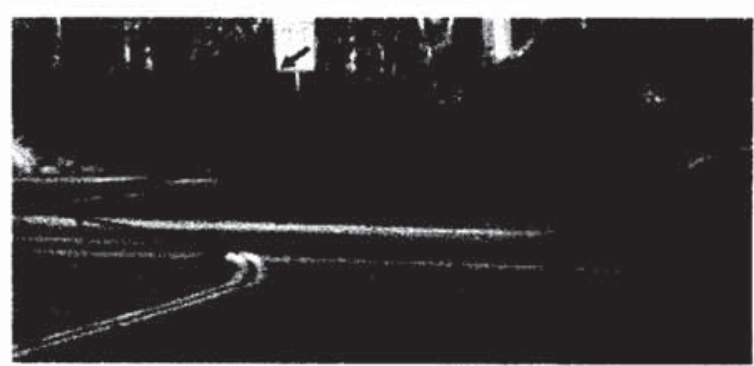

Figure 8. Testing track-roundabout

\section{RESULT}

The obtained result includes simulations and testing data which are combined interactively together to present vehicle dynamic and stability performance in order to give an insight into the HIS system's response. To study the interconnected mechanical and hydraulic systems (vehicle and HIS) under large steady maneuvering, the real time simulation methodology used here is similar to the hardware-in-loop test concept, but not typical.

Mathematical modeling and simulations can explore the real system's nature and help us understand the system's functions and relationships between sub-systems. Simulations can guide the experiment design, e.g., to optimize sensor deployment. It is costly to deploy sensors to measure pressure inside hydraulic cylinders, and simulation indicates there is no significant difference among different points in one circuit. Therefore, one measurement point is adequate for monitoring one circuit.

Simulations also can expand the boundary of experiment study, e.g., able to look into the system responses which can not be or can hardly be measured by sensors. For example, the flow rate inside hydraulic circuits is less accessible to measuring but can be monitored by real time simulation. Certainly the input used for the simulation has to come from experimental measurement in order to run the real time simulation, in this case, vehicle steering input is measured from experiment and used as simulation input, as shown in Figure 9. 


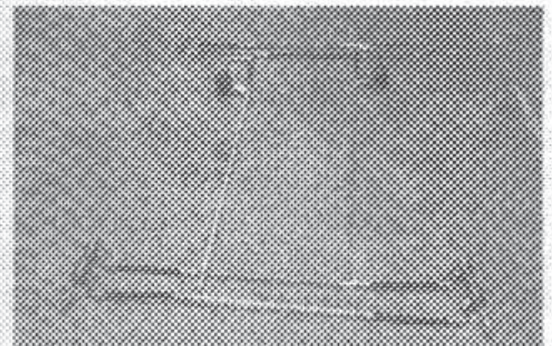

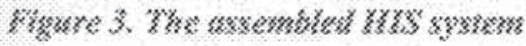

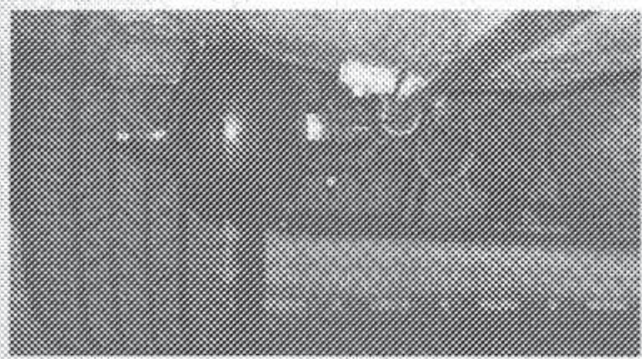

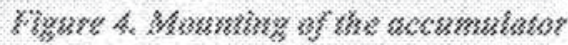

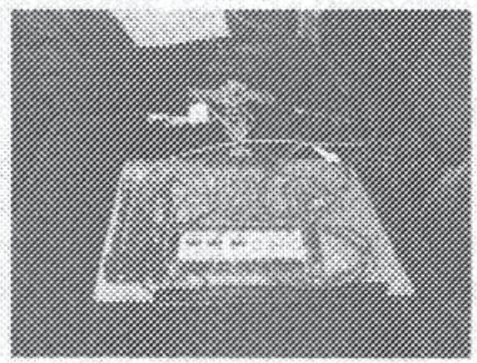

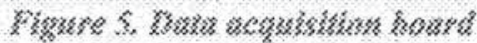

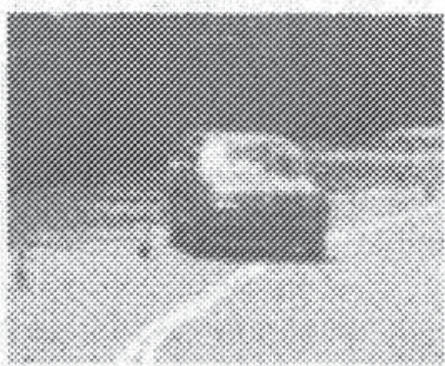

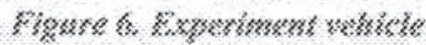

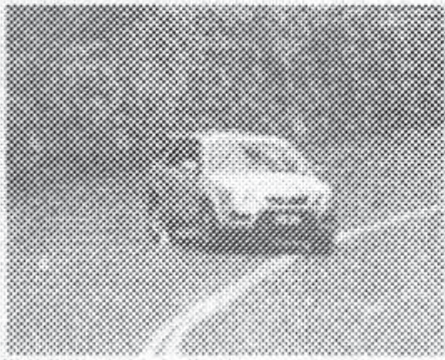

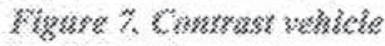

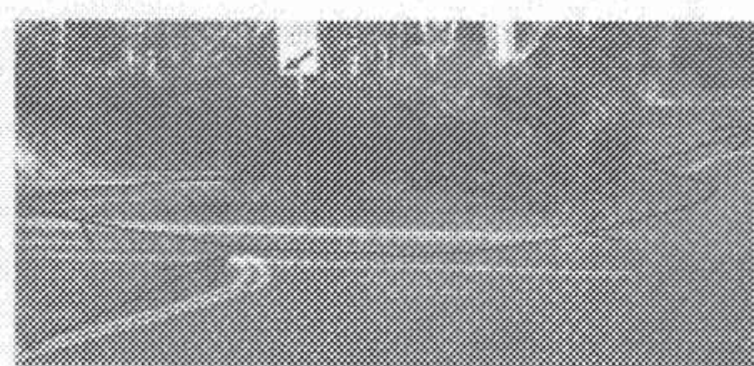

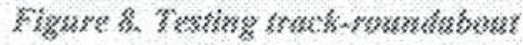

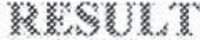

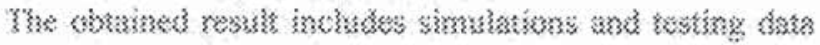

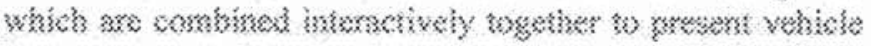

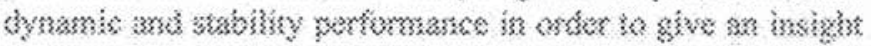

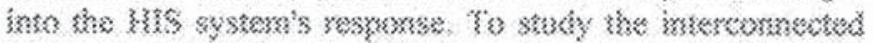

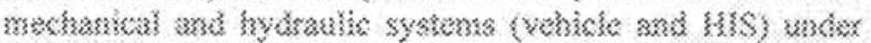

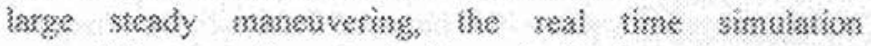

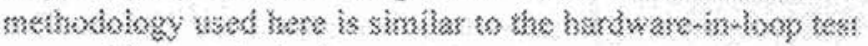

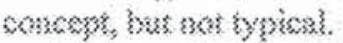

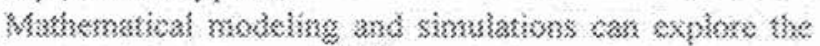

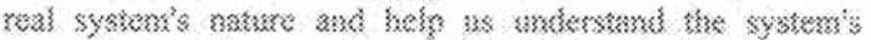

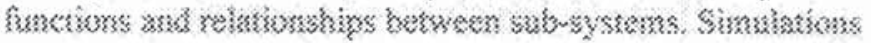

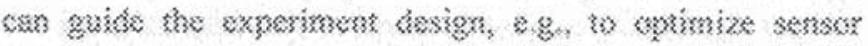

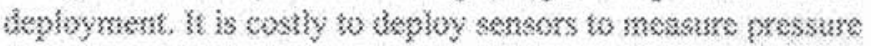

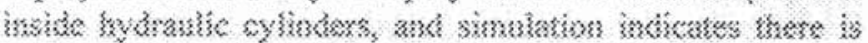

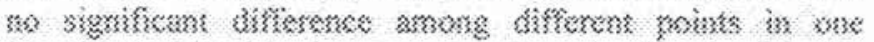

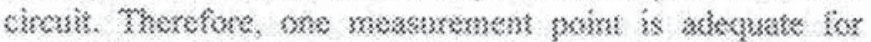

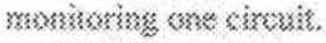

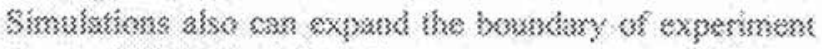

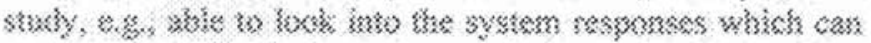

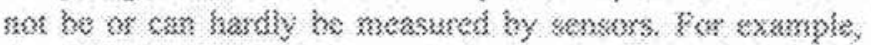

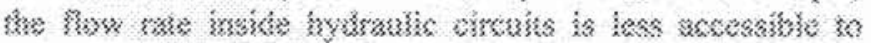

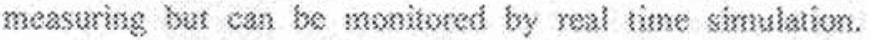

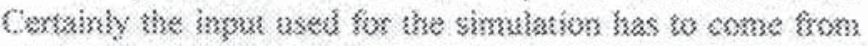

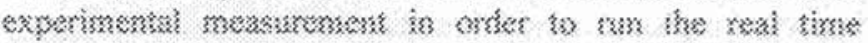

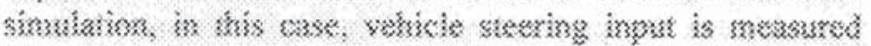

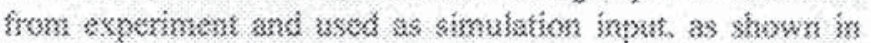
Gioure?. 


\section{SIMULATION INPUT}

To verify the simulation with this input signal, the simulated vehicle lateral acceleration is compared with testing data in Figure 10. It indicates the steering input that is estimated from tests is of good accuracy to be used as real time simulation input.

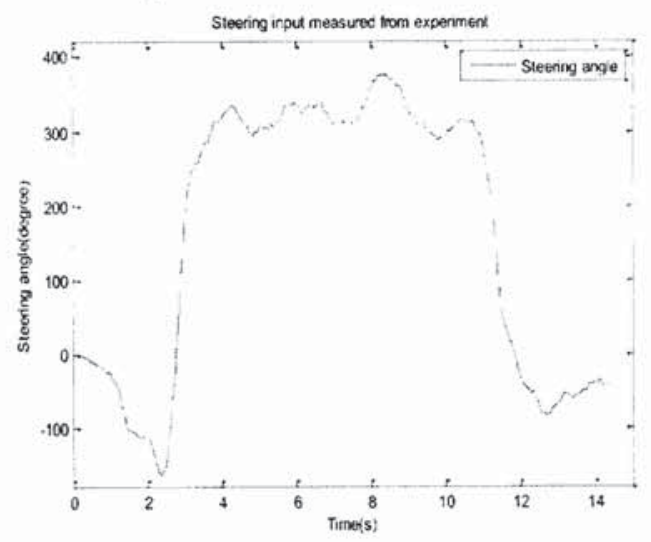

Figure 9. Steering input

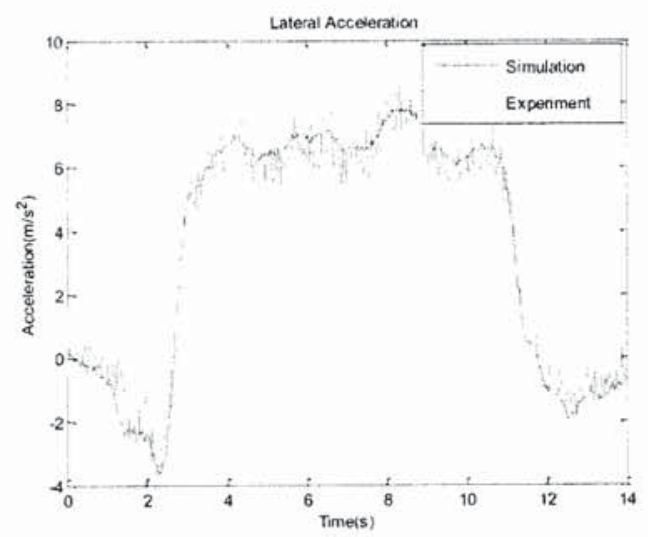

Figure 10. Vehicle lateral acceleration

\section{ROLL}

Data from three testing vehicles are used in the following sections, to keep simple and avoid confusion three cars are named as following: the experiment vehicle fitted with the HIS system pre-charged with 0 bar pressure is referred to " 0 bar HIS car", the experiment vehicle fitted with the HIS system pre-charged with 40 bar pressure is referred to " 40 bar HIS car", and the conventional vehicle with standard anti-roll bars is called "anti-roll bar car".

The vehicle roll performance certainly is the most important aspect to assess these two anti-roll systems. Figure 11 compares the performance of hydraulic anti-roll system with anti-roll bars. The vehicle body roll angle is estimated from suspension travels, so this roll angle is smaller than the real vehicle roll angle since the tire deflection is not included. The dotted blue line is the experimental car with a HIS system pre-charged with Obar, which means HIS is de- functioned and represent a car with no anti-roll systems. Thus the largest vehicle roll motion is expected and witnessed from the graph. This data is used as benchmark to evaluate other anti-roll systems. The dashed black line refers to the standard car with anti-roll bars and it proves the effectiveness of antiroll bars in vehicle roll control. The solid red line refers to the experimental car fitted with the $40 \mathrm{bar}$ pre-charged HIS system, and it shows an obvious advantage of HIS over antiroll bars in minimizing the vehicle roll angle.

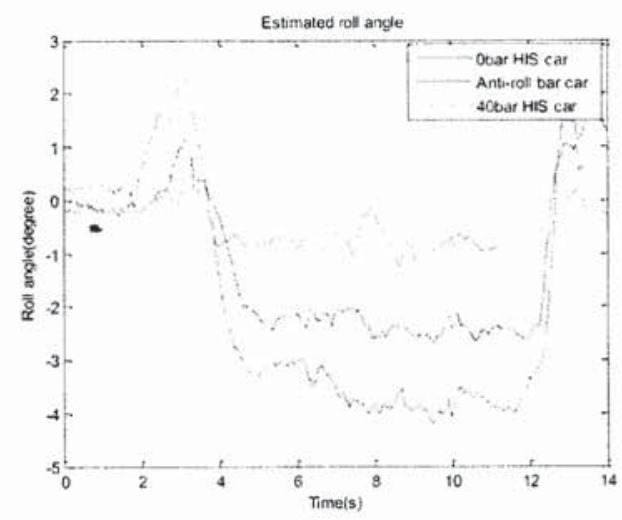

Figure 11. Vehicle roll angle calculated from suspension travel

\section{VERTICAL}

Vehicle vertical response is closely associated with the ride comfort and is critical to the evaluation of an anti-roll system. Both anti-roll bars and HIS systems are working in vehicle roll plane, and should not affect vehicle vertical motion. However, road tests are necessary to be conducted to prove this. Figure 12 shows the vertical displacement of three testing vehicles during the maneuver, and they approximately overlap each other during the turning period. No noticeable difference can be spot from these three cars in terms of vertical displacement, and this indicates HIS system might have no effect to vehicle bounce mode. To future prove it, vertical acceleration is provided for comparison, because the vertical acceleration is the most important index that related to the ride comfort. Figure 13 shows the three acceleration measurements taken from the three cars in time domain, and Figures 14 and 15 show the comparison of them in frequency domain. 40 bar HIS car slightly has an lower energy level in frequency domain compare to 0 bar HIS car and anti-roll bar car. The reason for that might be it slightly adds some damping effect to the bounce mode, due to the fluid viscosity. And this damping effect seems growing with the increased pre-charged system pressure. The RMS (root mean square) of the data in Figure 13 are: $10.117910 .113810 .1081\left(\mathrm{~m} / \mathrm{s}^{2}\right)$, which match the observation. 


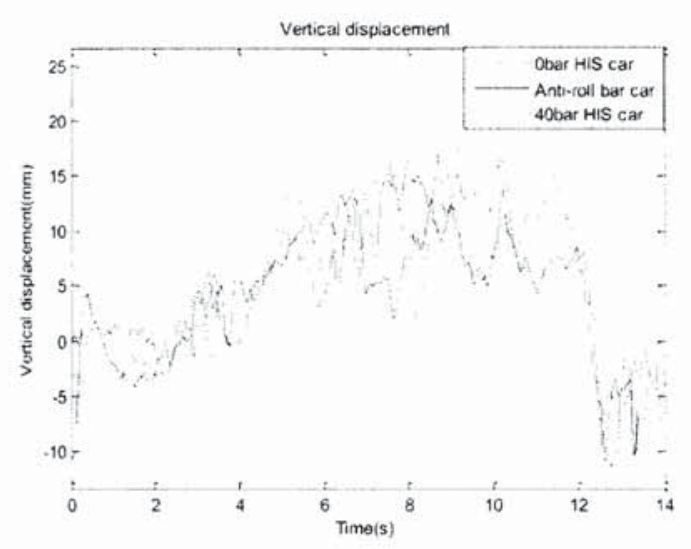

Figure 12. Estimated vehicle vertical displacement

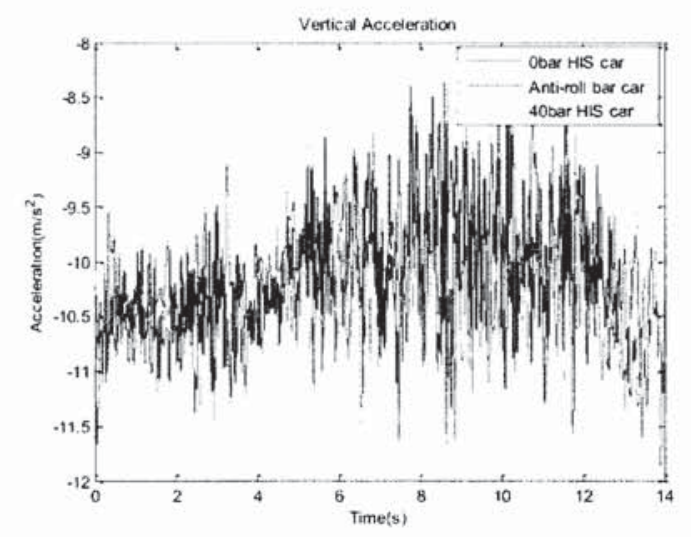

Figure 13. Measured vehicle vertical acceleration

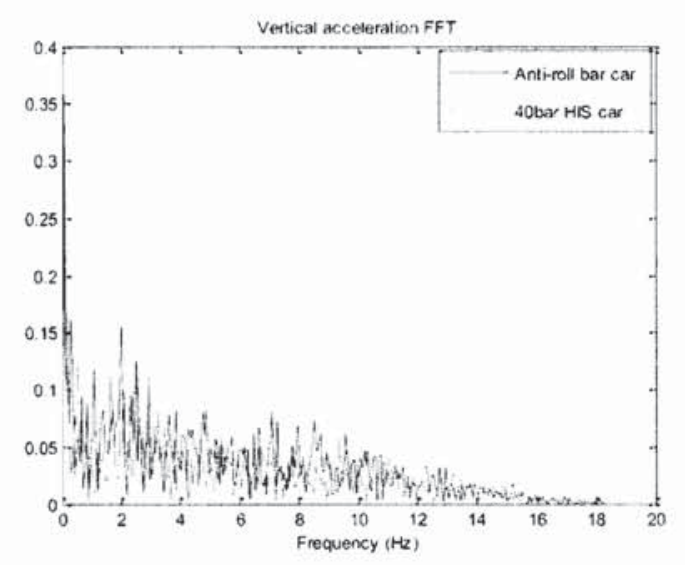

Figure 14. Vertical acceleration in FFT 1

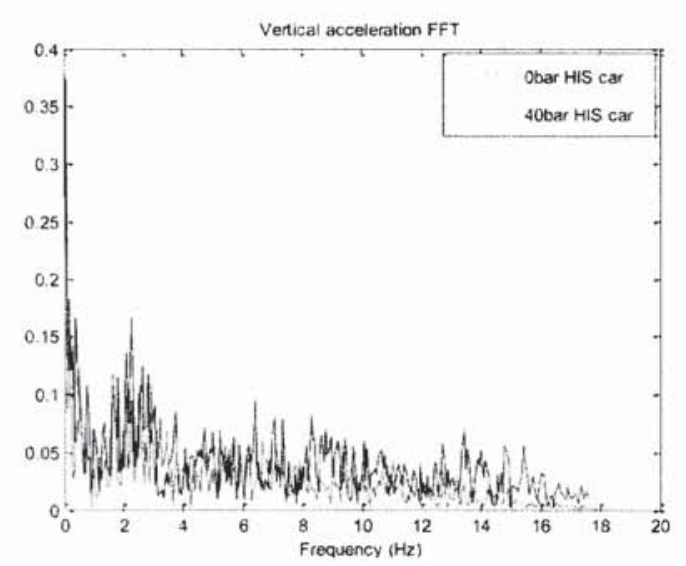

Figure 15. Vertical acceleration in FFT 2

\section{LATERAL}

Lateral motion is important and may lead the vehicle out of control. Once the centrifugal force that a vehicle experiences during cornering exceeds the friction force that its tire/ground contact can generate, sliding and yaw motions occur. This section investigates the lateral acceleration of the vehicles fitted with anti-roll bars and HIS systems respectively.

Figure 16 and Figure 17 compare the lateral acceleration of the three testing cars. Since the lateral acceleration is generated by centrifugal force, anti-roll system should not have direct affect to the average value of lateral accelerations. By increasing the pre-charged pressure from 0 bar to 40 bar, Figure 16 shows no noticeable difference. But from the comparison of anti-roll bars and HIS system in Figure 17, the difference is significant and interesting. The average values of these two curves still remain similar but the vehicle with anti-roll bars experiences significant oscillation around the peak lateral acceleration. The possible reason is that the lateral motion is coupled with vehicle roll motion and thus the installed anti-roll systems may have effects on vehicle lateral motion control. Both anti-roll bars and HIS can suppress roll motion, as showed above, but through different mechanism. The oscillation in lateral acceleration might due to the lack of damping of anti-roll bars, or the kinematic restrain that anti-roll bars brought to suspension. More research is required to explain/understand this phenomenon. 


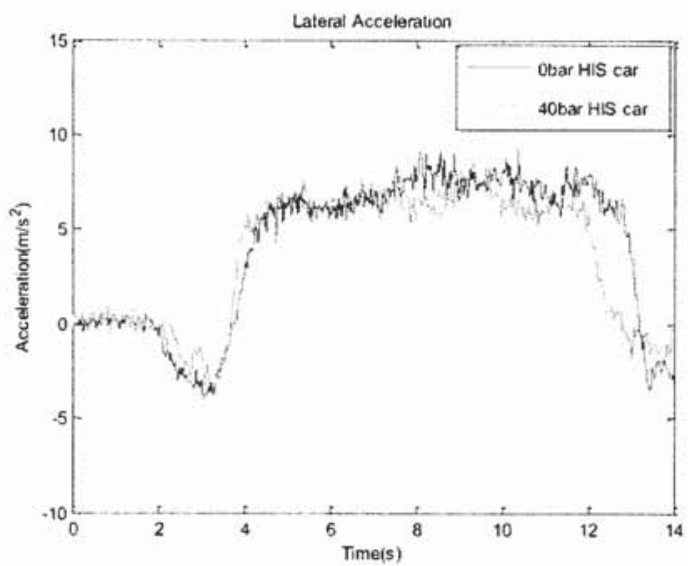

Figure 16. Lateral acceleration comparison 1

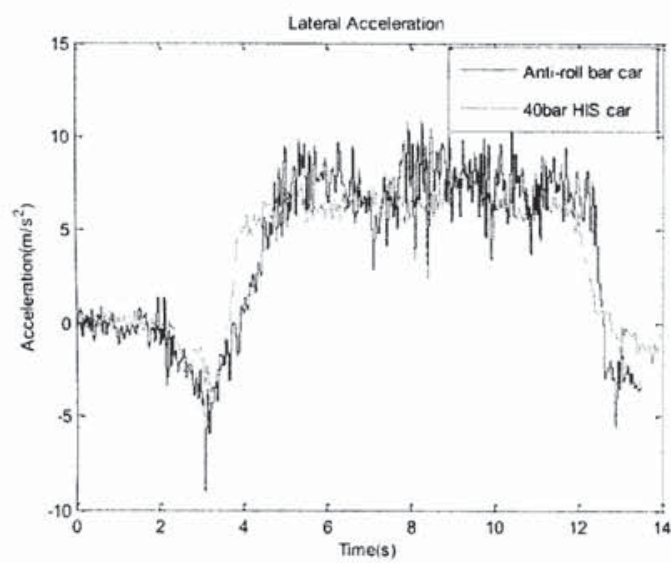

Figure 17. Lateral acceleration comparison 2

\section{HYDRAULIC SUSPENSION}

Experimental studies of hydraulic suspension response during the extreme maneuver are of great value to the system design. Here the simulation and experiment data are used together to give a complete picture of the 40 bar pre-charged HIS system's response under constant steering input. Two pressure sensors are installed into the front left/right joint points A and E (Figure 2) of HIS system, and the pressure data provided in Figure 18 and $\underline{19}$ is compared with simulations. The HIS system is pre-charged with 40 bar when no payload is on board. However, the system pressure rises to 47 bar after loading two testing personal and equipments. This change is considered in simulation. From Figure 18 and $\underline{19}$, the left side HIS pressure rising response matches simulation better than the right side, because in this test the right side accumulator was charged lower than what we required.

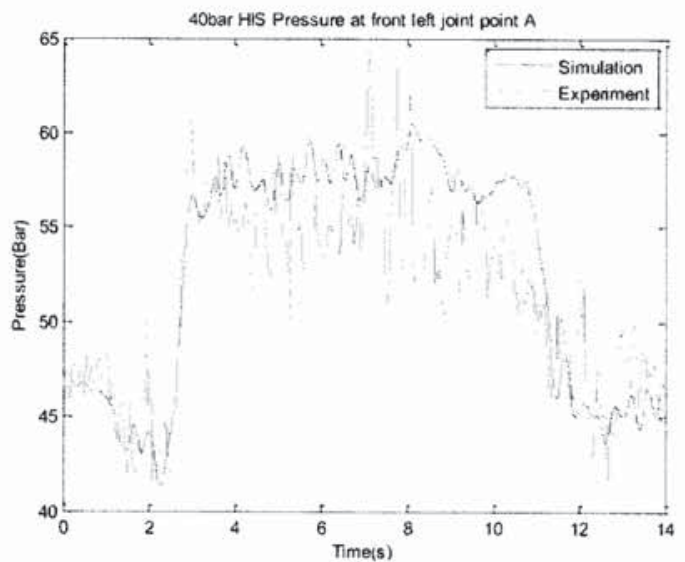

Figure 18. Pressure in left circuit

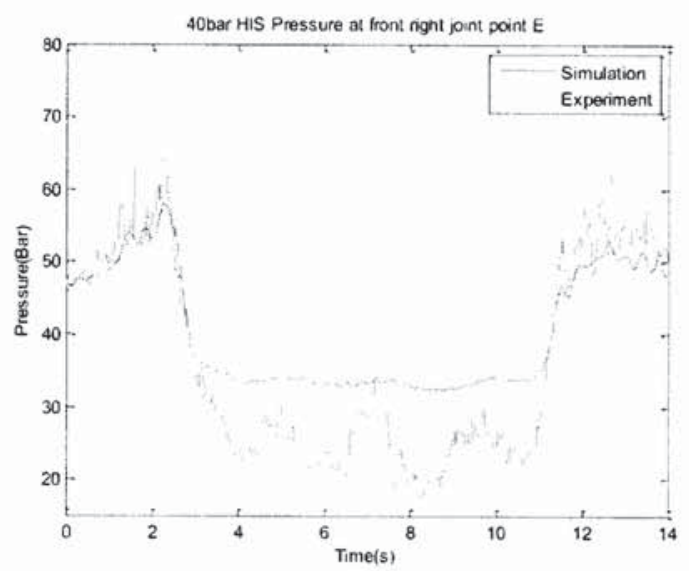

Figure 19. Pressure in right circuit

Figure 20 presents the pressure response and flow rate at different points of hydraulic circuits, as well as the total flow change in the accumulator. From the pressure response side, different points in one circuit share very similar characteristics in the pressure response, hence one measurement point from once circuit should be sufficient for us to monitor the system response. Different from pressure response, the flow rate vary from point to point. 

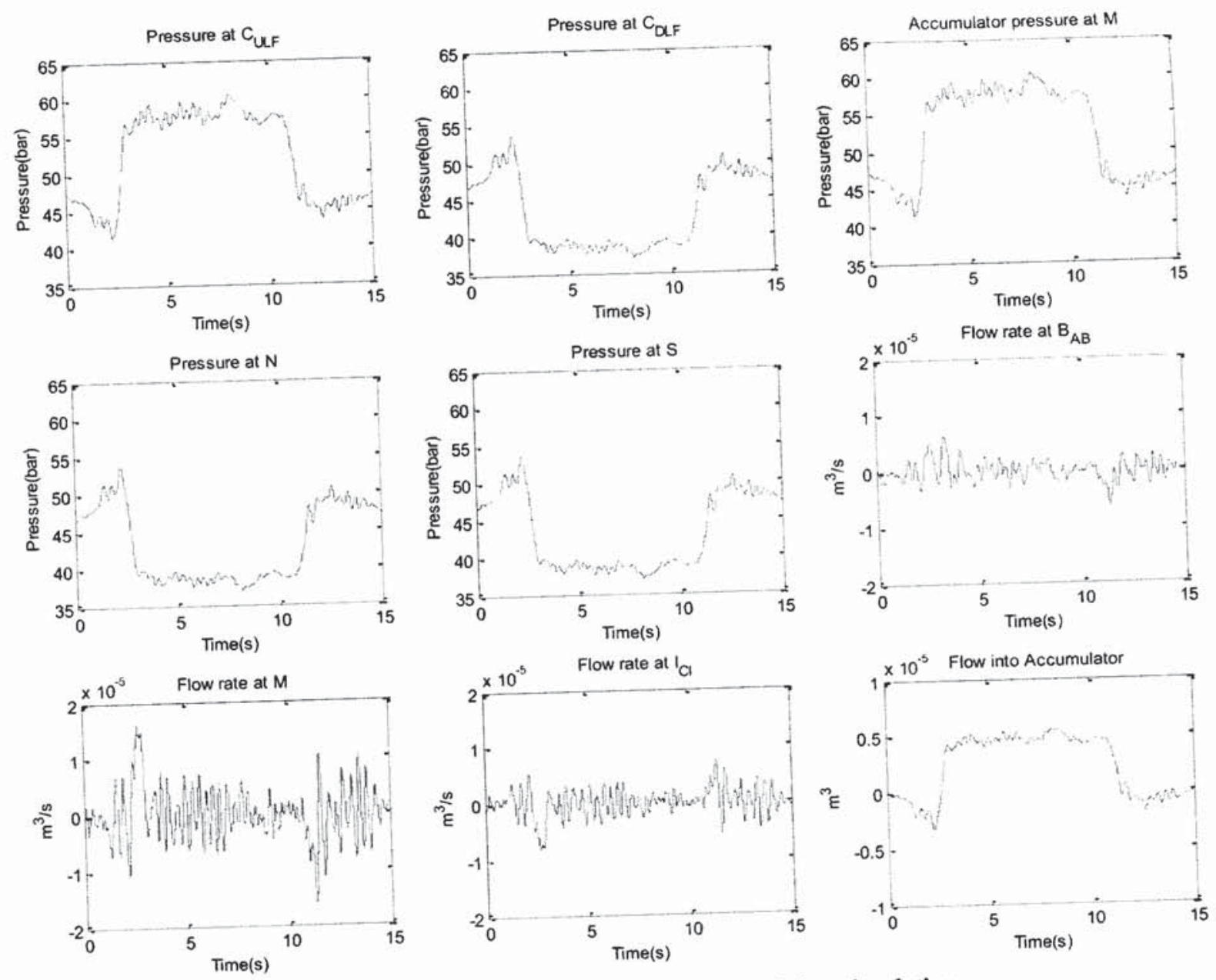

Figure 20. Hydraulic suspension system real time simulation

\section{CONCLUSION}

This paper presents an experimental investigation of the ffects that a hydraulically interconnected suspension brings to a vehicle. Vehicle dynamic performance in roll, vertical, ateral direction has been assessed based on testing data collected from a specified constant steering maneuver. A conventional vehicle fitted with an anti-roll bar is used for comparison in the study. It has been found that the hydraulically interconnected suspension has superior anti-roll ability over anti-roll bar, and does not affect ride comfort in vertical motion. One interesting finding learned from the hateral acceleration study is that the HIS system can significantly suppress the lateral oscillation that otherwise exists in the vehicle fitted with an anti-roll bar. The inherent connection between anti-roll systems lateral motion is not very clear and should be further investigated in future.

\section{ACKNOWLEDGMENTS}

The financial support of this work by the Australian Research Council (ARC DP0988429) and the University of Technology, Sydney, is gratefully acknowledged.

\section{REFERENCES}

Smith, M. \& Walker, G. 2005, 'Interconnected vehicle suspension', Proceedings of the Institution of Mechanical Engineers, Part D: Journal Procedingsile Engineering, vol. 219, no. 3, pp. 295-307.

of Automobile Engineering, vol. 219, no. 3, pp. 295-307-

2. Cao, D., Rakheja, S. \& Su, C.-Y. 2010, Roll- and pitch-plane coupled hydro-pneumatic suspension - Part 1: Feasibility antional Journal suspension properties', Vehicle System Dynamics: International of Vehicle Mechanics and Mobility, vol. 48, no. 3, pp. 361-86.

. 'Balanced Suspension', SAE paper 2000-01-3572

3. Zapletal, E. 200, "Integral Suspension System for Motor Vehicles

. Fontdecaba i Buj, J., "Integral Suspents 2002, doi: $10.4271 / 2002-01-3105$.

5. Rauh, J. \& Ammon, D. 2011, 'System dynamics of electrified vehicles: some facts, thoughts, and challenges', Vehicle System Dynamics, vol. some facts, thoughts, $1005-20$.

6. Zhang. N Wang \& 4 Hu , 2011, 'Handling analyses of a Vehicle Fitted with a Roll-resistant Hydraulically Interconnected Suspension', paper presented to the IAVSD, Manchester, UK

dynamics, and control', Vehicle System Dynamics, vol. 49, no. 1-2, pp. 3-28.

8. Smith, W 2009, 'An investigation into the dynamics of vehicles with hydraulically interconnected suspensions', Ph.D thesis, University of Technology, Sydney.

. The Lovins, A.B. \& Cramer, D.R. 2004, 'Hypercars no. 1-2, pp. 50-85.

10. Smith W. A. Zhang N. \& Hu, W. 2010, 'Hydraulically interconnected 年, vehicle suspension. harnal of Vehicle Mechanics and Mobility. International Journal of Vehicle Mechanics and Mobility. 'Hydraulically 11. Smith, W.A., Zhang, N. \& Jeyakumaran, J. 2010, 'Hydraulically 
analysis', Vehicle System Dynamics: International Journal of Vehicle Mechanics and Mobility, vol. 48, no. 1, pp. 41-64.

12. Zhang, N., Smith, W.A. \& Jeyakumaran, J. 2010, 'Hydraulically interconnected vehicle suspension: background and modelling', Vehicle System Dynamics: International Journal of Vehicle Mechanics and Mobility, vol. 48 , no. 1 , pp. $17-40$

13. Wilde, J., Heydinger, G., Guenther, D., Mallin, T. and Devenish, A., "Experimental Evaluation of Fishhook Maneuver Performance of a Kinetic Suspension System," SAE Technical Paper 2005-01-0392, 2005 doi: $10.4271 / 2005-01-0392$.

14. Cao, D., Rakheja, S. \& Su, C.-Y. 2010, 'Roll- and pitch-plane-coupled hydro-pneumatic suspension. Part 2: dynamic response analyses', Vehicle System Dynamics: International Journal of Vehicle Mechanics and Mobility, vol 48, no. 4, pp. 507-28.

15. Cao, D., Rakheja, S. \& Su, C.-Y. 2006, 'A Generalized Model of a Class of Interconnected Hydro-Pneumatic Suspensions and Analysis of Pitch Properties', ASME Conference Proceedings, vol. 2006, no. 47675, pp. 137-46.

16. Cao, D., Rakheja, S. \& Su, C.Y. 2007, 'Roll plane analysis of a hydropneumatic suspension with twin-gas-chamber struts', International Journal of Heavy Vehicle Systems, vol. 14, no. 4, pp. 355-75.

17. Cao, D., Rakheja, S. \& Su, C.Y. 2008, 'Dynamic analyses of heavy vehicle with pitch-interconnected suspensions', International Journal of Heavy Vehicle Systems, vol. 15 , no. 2-4, pp. 272-308

18. Mace, N. 2004, 'Analysis and synthesis of passive interconnected vehicle suspensions', PhD thesis thesis, Cambridge University.

19. Cao, D., Rakheja, S. and Su, C.-Y., "Comparison of Roll Properties of Hydraulically and Pneumatically Interconnected Suspensions for Heavy Vehicles," SAE Technical Paper 2005-01-3593, 2005, doi: 10.4271/2005-01-3593.

20. Cao, D., Rakheja, S. \& Su, C.Y. 2008, 'Pitch plane analysis of a twingas-chamber strut suspension', Proceedings of the Institution of Mechanical Engineers, Part D: Journal of Automobile Engineering, vol. 222 , no. 8 , pp. $1313-35$.

21. Wilde, J., Heydinger, G. and Guenther, D., "ADAMS Simulation of Ride and Handling Performance of Kinetic Suspension System," SAE Technical Paper 2006-01-1972, 2006, doi:10.4271/2006-01-1972.

22. Zhang, N. Wang L. Hu W \& Ding F 2010 Dynamic Characteristics of a Vehicle Fitted with a Roll-Resistant Hydraulically Interconnected Suspension', paper presented to the The First IFToMM Asian Conference on Mechanism and Machine Science, Taipei, Taiwan, October $21-25,2010$

23. Savkoor, A.R., Happel, H. \& Horkay, F. 1999, 'Vehicle handling and sensitivity in transient maneuvers', in Pauwelussen, J.P. (ed.), Vehicle performance: understanding human monitoring and assessment Swets\&Zeitlinger Publishers, Lisse, The Netherlands, pp. 121-47.

24. Hegazy, S., Rahnejat, H \& Hussain, K. 2000, 'Multi-Body Dynamics in Full-Vehicle Handling Analysis under Transient Manoeuvre', Vehicle System Dynamics: International Journal of Vehicle Mechanics and Mobility, vol. 34 , no. 1 , pp. 1-24. 